\title{
Change only updating the 1:25 000 Swiss National Map
}

\author{
Dominik Käuferle $^{\mathrm{a}}$, Mark Wigley ${ }^{\mathrm{b}}$ \\ ${ }^{a}$ Federal Office of Topography swisstopo,Dominik.kaeuferle@swisstopo.ch \\ ${ }^{b}$ Esri SchweizAG,m.wigley@esri.ch
}

Keywords: Topographic maps, change only updates, incremental updates, Swiss National Maps, ArcGIS

\section{Abstract:}

The Swiss National Map series including its production processes has been completely revised between 2001 and 2020. In the past the "old map", created between 1952 and 1979, was produced using glass engraving techniques and later updated using a CAD system. In contrast, the "new map" was created directly from a high-resolution GIS topographic landscape model, the TLM. The TLM was used to create a number of GIS cartographic models, so-called digital cartographic models (DCM) - one per national map scale - using a variety of automatic generalisation procedures and interactive workflows. The production of the DCMs started in 2013 and the initial creation of each DCM was complete in 2020. Thanks to a high degree of automation, it was possible to decrease the manual work by over $20 \%$ compared to the CAD updates. The DCMs are implemented as ArcGIS geodatabases and the cartographic production system Genius-DB is used as a custom ArcMap application for the production.
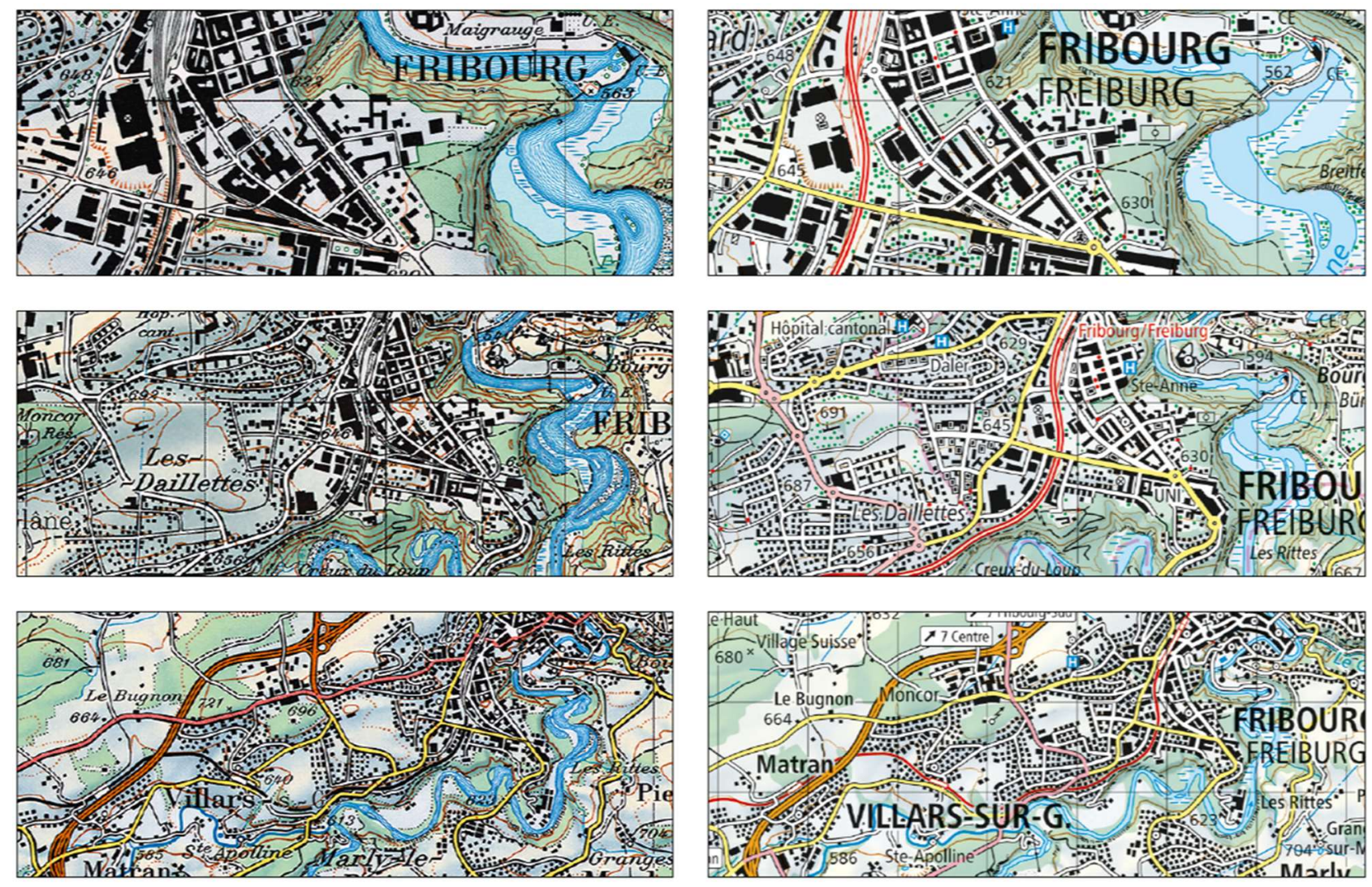

Figure 1. Left: "old" Swiss National Maps 1:25 000, 1: 50 000, 1:100 000 before 2013. Right: corresponding "new" Swiss National Maps, produced as digital cartographic Models DCM

Swisstopo completely updates all map sheets at least every six years, depending on updates in the TLM. Updating the first 1:25 000 map sheets started in early 2021. These updates focus on only the changes in the base data (the TLM) so as to preserve as much of the interactive cartographic work as possible. Hence areas unchanged in TLM stay unchanged in the DCM's, unless necessary for cartographic reasons. These are called "change only updates" or "incremental updates". If an area needs to be updated, this is what is done: 
1. Compare the old and the new state of TLM in order to build the increment. The increment can be an addition, a modification, or a deletion. (automatic)

2. Integrate the changes into a copy of the DCM, which is to insert, update or delete the corresponding DCM features (partly automatic, mainly manual)

3. Cartographically correct the area directly surrounding the changed features (manual)

In order to offer the cartographers the optimal support, swisstopo has implemented new workflows and has added new tools to the existing production system Genius-DB. As of May 2021, these workflows and tools are in production for updating the first 12 1:25000 National Map sheets. At the ICC, swisstopo will present the experiences gained, over the course of the last 7 months, in working with this new update methodology. 